\title{
Activating metabotropic glutamate receptor-7 attenuates visceral hypersensitivity in neonatal maternally separated rats
}

\author{
LIMEI SHAO $^{1 *}$, YANBING LIU $^{2^{*}}$, JUNHUA XIAO $^{3}$, QUNYING WANG $^{1}$, FEI LIU $^{2 *}$ and JIN DING ${ }^{1^{*}}$ \\ ${ }^{1}$ Department of Gastroenterology, Jinhua Hospital of Zhejiang University, Jinhua, Zhejiang 321000; \\ ${ }^{2}$ Department of Gastroenterology, Shanghai East Hospital, Tongji University, Shanghai 200092; ${ }^{3}$ Department of \\ Gastroenterology, The First Affiliated Hospital of Soochow University, Suzhou, Jiangsu 215000, P.R. China
}

Received February 24, 2018; Accepted December 7, 2018

DOI: $10.3892 / \mathrm{ijmm} .2018 .4022$

\begin{abstract}
Increasing evidence has indicated that metabotropic glutamate receptor-7 (mGluR7) is an important target for reducing anxiety and stress-associated behaviours. Notably, mood disorders exhibit high levels of comorbidity with gastrointestinal dysfunction; however, the role of mGluR7 outside of the central nervous system is currently unknown. Activating mGluR7 likely increases colonic secretory function. Therefore, the present study aimed to evaluate the possible effects of mGluR7 on the visceral hypersensitivity of irritable bowel syndrome (IBS) in rats. The expression levels of mGluR7 were assessed in the colon tissues of rats with neonatal maternal separation (NMS)-induced visceral hypersensitivity using reverse transcription-quantitative polymerase chain reaction, western blotting and immunohistochemistry. In addition, the mGluR7 agonist AMN082 (3 or $10 \mathrm{mg} / \mathrm{kg}$; i.p.) was administered $1 \mathrm{~h}$ prior to the visceral hypersensitivity test, and the effects of AMN082 were then observed on the nuclear factor $(\mathrm{NF})-\kappa \mathrm{B}$ signalling pathway. The mRNA and protein expression levels of mGluR7 were upregulated in the colon mucosa of NMS rats compared with in normal control rats. Notably, administration of AMN082 $(10 \mathrm{mg} / \mathrm{kg})$ attenuated colorectal distension (CRD)-induced visceral hypersensitivity in NMS rats. In addition, interleukin-10 and transforming growth factor- $\beta$ mRNA expression levels were upregulated, whereas
\end{abstract}

Correspondence to: Dr Fei Liu, Department of Gastroenterology, Shanghai East Hospital, Tongji University, 150 Jimo Road, Shanghai 200092, P.R. China

E-mail: doctorfeiliu@163.com

Dr Jin Ding, Department of Gastroenterology, Jinhua Hospital of Zhejiang University, 351 Mingyue Road, Jinhua, Zhejiang 321000, P.R. China

E-mail:45338363@qq.com

*Contributed equally

Key words: irritable bowel syndrome, neonatal maternal separation, visceral hypersensitivity, mGluR7, inflammatory factors, nuclear factor- $\kappa \mathrm{B}$ interferon- $\gamma$ mRNA expression levels were downregulated in the NMS + AMN082 group compared with in NMS rats. The number of cluster of differentiation $3^{+} \mathrm{T}$ cells in the intestinal mucosa and myeloperoxidase activity were decreased in NMS + AMN082 rats. Furthermore, AMN082 treatment reduced the protein expression levels of phosphorylated-NF- $\kappa \mathrm{B}$ in the colon tissue of NMS rats. These results indicated that activation of mGluR7 may attenuate CRD-induced visceral hypersensitivity in experimental IBS and reduce the abnormal immune cytokine response. In addition, it was suggested that the role of AMN082 in modulating the inflammatory response may be partially associated with inhibiting NF- $\mathrm{kB}$ activation. These data suggested that targeting mGluR7 may be useful in the treatment of stress-associated IBS.

\section{Introduction}

Irritable bowel syndrome (IBS) is a highly prevalent functional bowel disorder, which is characterised by the presence of abdominal pain or discomfort, an alteration in bowel habits, and diarrhoea and constipation without any structural cause. The aetiology of IBS predominantly includes genetics, motility, visceral hypersensitivity, diet, infection and inflammation, alteration of intestinal flora and psychosocial factors $(1,2)$. Neonatal maternal separation (NMS) is a form of early-life stress, which has been consistently used in rats to develop experimental visceral hypersensitivity $(3,4)$. Visceral hypersensitivity has been widely considered a biological marker of IBS; however, the underlying mechanism remains unclear.

L-Glutamate is the major excitatory neurotransmitter in the central nervous system. The actions of glutamate are modulated by ionotropic receptors and metabotropic glutamate receptors (mGluRs) (5). Based on sequence homology and signal transduction mechanisms, mGluRs have been divided into groups I, II and III; mGluR4, mGluR6, mGluR7 and mGluR8 are group III mGluRs $(5,6)$. These receptors can modulate the effect and release of glutamate in the central nervous system (7). In addition, increasing evidence has indicated that mGluRs are expressed in the periphery, such as the gastrointestinal system. The glutamatergic system has also been implicated in the pathophysiology of depression and anxiety; antidepressants have been reported to inhibit glutamate release, a phenomenon mirrored by activation of group III 
mGluRs $(6,8)$. As a member of the group III mGluRs, mGluR7 is of particular interest because it is an important target for reducing anxiety and stress-associated behaviours $(8,9)$. Selective activation of G-protein-coupled mGluR7 elicits anxiolytic-like effects in mice by modulating $\gamma$-aminobutyric acid-ergic neurotransmission (10). Furthermore, activating mGluR7 by AMN082 (a selective agonist of mGluR7) induces a reduction in immobility in a forced swim test and tail suspension test $(11,12)$. Notably, mood disorders exhibit high comorbidity with gastrointestinal dysfunction (13), and a high frequency of IBS symptoms is observed in patients with panic disorder, generalised anxiety disorder and major depressive disorder (14). Therefore, dysregulation of mGluR7 activity may be considered a significant factor underlying stress-induced IBS. AMN082 is a recently discovered selective mGluR7 agonist, which induces an increase in faecal water content in stress-induced defecation (6); however, to the best of our knowledge, the contribution of mGluR7 to early-life stress-induced visceral hypersensitivity in IBS remains unexplored. Therefore, the present study aimed to investigate a possible functional role of mGluR7 in the colon by assessing agonist-induced alterations in visceral hypersensitivity.

Several 5-hydroxytryptamine receptors (5-HTRs) are expressed in the gut, including $5-\mathrm{HT}_{1 \mathrm{~A}} \mathrm{R}, 5-\mathrm{HT}_{2 \mathrm{~A}} \mathrm{R}, 5-\mathrm{HT}_{2 \mathrm{~B}} \mathrm{R}$, $5-\mathrm{HT}_{3} \mathrm{R}, 5-\mathrm{HT}_{4} \mathrm{R}$ and $5-\mathrm{HT}_{7} \mathrm{R}$. Alterations in the levels of 5-HT have been observed in experimental models of colitis and in patients with IBS; however, the results are varied $(15,16)$. Nitric oxide (NO) is a gaseous messenger that serves an essential role in the physiology and pathophysiology of the gastrointestinal tract. NO is synthesised by NO synthase (NOS), which is classified into neuronal NOS, endothelial NOS and inducible NOS (iNOS). It has been reported that activation of the NO pathway alleviates the symptoms of IBS; Paragomi et al demonstrated that the NOS inhibitor L-NAME reverses the antinociceptive effects of sodium hydrogen sulphide on colorectal distension in a chemically induced model of IBS in rats (17). These findings indicated that $\mathrm{NO}$ may serve a protective role against IBS; however, the expression levels of NO in the rectum and plasma in IBS are not consistent $(3,17)$. In addition, the development of IBS is associated with low-grade inflammation, and nuclear factor (NF)- $\mathrm{\kappa B}$ is a critical transcription factor for the inflammatory response (18). Anxiety-depression status may elevate interleukin (IL)-1 $\beta$ and IL-10 levels in patients with IBS (19), which leads to the occurrence or aggravation of IBS. The present study demonstrated that mGluR7 may serve an important role in visceral hypersensitivity by modulating the function of NOS or inflammatory factors, thereby attenuating visceral hypersensitivity in IBS. The results indicated that there was no difference in 5-HTRs in any of the groups; therefore, alterations in NOS and inflammatory factors were discussed in detail.

\section{Materials and methods}

Animals and NMS. All animal protocols were approved by the Animal Care and Use Committee at the Tongji University School of Medicine (Shanghai, China) and were conducted according to the National Institutes of Health (NIH) Guidelines for the Care and Use of Animals in Research (NIH Publication No. 85-23, revised 1996) (20). Seven 15-day pregnant Sprague-Dawley rats were obtained from Shanghai SLAC Laboratory Animal Co., Ltd. (Shanghai, China). The rats were maintained under a 12-h light/dark cycle with free access to food and water at $22^{\circ} \mathrm{C}$ and $50 \%$ relative humidity.

All Sprague-Dawley pups (weight 5-6 g) were divided into three groups: Normal control (NC) group, neonatal maternal separation (NMS) group and NMS + AMN082 group ( $\mathrm{n}=6 /$ group). The pups were maintained under a 12 -h light/dark cycle with free access to food and water at $22^{\circ} \mathrm{C}$ and $50 \%$ relative humidity. Pups in the NMS and NMS + AMN082 groups were separated from their dams and placed into individual cages in an adjacent room for $3 \mathrm{~h}$ (09:00-12:00) on postnatal day (P)2-14 (date of birth is designated P0). The pups were subsequently returned to the cages of their dams for the remaining time. The rats in the $\mathrm{NC}$ group were allowed to remain in standard cages with their dams. Pups were weaned on P22; only male rats were used in the present study, in order to avoid alterations associated with hormonal cycles. Each cage housed five rats; visceral hypersensitivity was measured after 8 weeks of feeding after weaning.

Behavioural testing to measure visceral hypersensitivity Abdominal withdrawal reflex (AWR). Visceral hypersensitivity responses to colorectal distension (CRD) were measured by recording AWR scores, as described previously $(3,10)$. Prior to testing, the rats were fasted and subjected to water deprivation, in order to reduce faeces. The rats were anaesthetized with isoflurane (1.5\%) in a sealed cage (21), and a $6-\mathrm{cm}$ deflated latex balloon was inserted into the distal colon up to $1 \mathrm{~cm}$ from the anal verge. After a 15-min recovery period, measurements of the visceral pain threshold were recorded when various pressure was applied to the abdominal walls by inflating the balloon (20,40, 60 and $80 \mathrm{mmHg}$ ). Three measurements for each animal were taken at 5-min recovery intervals. A previously reported standard was used to evaluate the AWR score: 0 , no response to given pressure; 1 , slight head movement without abdominal muscle contraction; 2 , contraction of the abdominal muscles; 3 , lifting of the abdominal wall; and 4 , body arching and lifting of pelvic structures (22).

Electromyography $(E M G)$. EMG was also used to quantitatively measure visceral hypersensitivity at week 8 . The surgical procedures for EMG were performed as previously described $(23,24)$. Rats were deeply anaesthetized with $30-40 \mathrm{mg} / \mathrm{kg}$ (3-4 mg/ml) 3\% sodium pentobarbital (i.p.), no signs of toxicity were observed. A pair of EMG electrodes was surgically implanted in the lower left abdominal area to expose the external oblique abdominal musculature, and the electrode was tunnelled subcutaneously, exteriorised and secured at the back of the neck for EMG recording. Rats were allowed to recover for $\geq 5$ days. Fasting and water deprivation, isoflurane anaesthesia and insertion of the deflated latex balloon were conducted as in the AWR test. Subsequently, the electrodes were connected to a BL-420F Data Acquisition \& Analysis system (Chengdu Techman Software Co., Ltd., Chengdu, China), and to record the EMG signal, various pressure was applied to the abdominal walls by inflating the balloon (20,40, 60 and $80 \mathrm{mmHg}$ ). The procedure was repeated three times and the EMG signals were recorded. Alterations in the EMG signal response to CRD were determined by calculating the 
Table I. Sequences of primers used for reverse transcription-quantitative polymerase chain reaction.

\begin{tabular}{|c|c|c|}
\hline Gene & Forward primer & Reverse primer \\
\hline IL-10 & 5'-agtggagcaggtgaagaatga-3' & $5^{\prime}$-cacgtaggcttctatgcagttg-3' \\
\hline TGF- $\beta 1$ & 5'-tggagcetggacacacagta-3' & 5'-tagtagacgatgggcagtgg-3' \\
\hline IFN- $\gamma$ & 5'-tcatcgaatcgcacctgat-3' & 5'-ggatctgtgggttgttcacc-3' \\
\hline iNOS & 5'-gagacaggaagtcggaagc-3' & 5'-gtgttgaaggcgtagctgaa-3' \\
\hline eNOS & 5'-tgtagctgtgctggcataca-3' & 5'-ttgagttggctcatccatgt-3' \\
\hline nNOS & 5'-tcattagcaatgaccgaagc-3' & 5'-aacattggaaagaccttggg-3' \\
\hline $5-\mathrm{HT}_{1 \mathrm{~A}} \mathrm{R}$ & 5'-gatctcgctcacttggctca-3' & 5'-aaagcgccgaaagtggagta-3' \\
\hline $5-\mathrm{HT}_{2 \mathrm{~A}} \mathrm{R}$ & 5'-aagccaccttgtgtgtgagt-3' & 5'-tgtggatggaccgttggaag-3' \\
\hline $5-\mathrm{HT}_{3} \mathrm{R}$ & 5'-atgactgctcagccatggga-3' & 5'-tggtggtggaagagggctat-3' \\
\hline $5-\mathrm{HT}_{4} \mathrm{R}$ & 5'-tggtttcggtgctggtgaatg-3' & 5'-tgatgctgtggtgagtaggaca-3' \\
\hline $5-\mathrm{HT}_{7} \mathrm{R}$ & 5'-atcacgagacccctcacgta-3' & 5'-tctgagcccatccgaagaga-3' \\
\hline mGluR7 & 5'-tgtgagccetgtgatggata-3' & 5'-ccagtttgatgattgggatg-3' \\
\hline GAPDH & 5'-agatccacaacggatacatt-3' & 5'-tccctcaagattgtcagcaa-3' \\
\hline
\end{tabular}

5-HT, 5-hydroxytryptamine; 5-HT ${ }_{1 \mathrm{~A}} \mathrm{R}, 5-\mathrm{HT} 1 \mathrm{~A}$ receptor; 5- $\mathrm{HT}_{2 \mathrm{~A}} \mathrm{R}, 5-\mathrm{HT} 2 \mathrm{~A}$ receptor; 5-HT $\mathrm{R}, 5-\mathrm{HT} 3$ receptor; 5-HT $\mathrm{R}_{4}$ 5-HT 4 receptor; 5 -HT 7 R, 5-HT 7 receptor; eNOS, endothelial nitric oxide synthase; IFN- $\gamma$, interferon- $\gamma$; IL-10, interleukin-10; iNOS, inducible nitric oxide synthase; mGluR7, metabotropic glutamate receptor-7; nNOS, neuronal nitric oxide synthase; TGF- $\beta 1$, transforming growth factor- $\beta 1$.

changes in the area under the curve (AUC) of raw EMG amplitude responses to $\mathrm{CRD}$, based on the formula $\triangle \mathrm{AUC}$ (AUC during CRD-AUC before CRD) $(3,21)$.

The selective mGluR7 agonist AMN082 (cat. no. 2385; Tocris Bioscience, Bristol, UK) was dispersed in a suspension of $0.5 \%$ methylcellulose (Sigma-Aldrich; Merck KGaA, Darmstadt, Germany) and was administered intraperitoneally (3 or $10 \mathrm{mg} / \mathrm{kg}$ ) to NMS rats in the NMS + AMN082 group $1 \mathrm{~h}$ prior to AWR and EMG testing. The same volume of PBS (vehicle control) was administered to rats in the NMS group. Doses were chosen based on previous experiments indicating behavioural and physiological alterations in response to this range $(6,25)$.

Tissue preparation. Rats were anaesthetized by $3 \%$ pentobarbital sodium (30 mg/kg i.p.) immediately following completion of the CRD study. The distal colons were dissected before the rats were sacrificed by decapitation. The colon samples were immediately frozen in liquid nitrogen, and stored at $-70^{\circ} \mathrm{C}$.

Reverse transcription-quantitative polymerase chain reaction $(R T-q P C R)$. Total RNA was extracted from the colon tissues using TRIzol ${ }^{\circledR}$ reagent (Invitrogen; Thermo Fisher Scientific, Inc., Waltham, MA, USA), according to the manufacturer's protocol. RNA was reverse-transcribed to cDNA using a PrimeScript $^{\mathrm{TM}}$ RT reagent kit (Takara Bio, Inc., Otsu, Japan), according to the manufacturer's protocol. mRNA transcripts were analysed by qPCR using SYBR ${ }^{\circledR}$ Premix Ex Taq ${ }^{\mathrm{TM}}$ (Takara Bio, Inc.) on an Applied Biosystems StepOne/StepOnePlus Real-Time PCR system (Applied Biosystems; Thermo Fisher Scientific, Inc.). qPCR was performed for 40 cycles, as follows: Initial denaturation at $95^{\circ} \mathrm{C}$ for $10 \mathrm{~min}$, denaturation at $95^{\circ} \mathrm{C}$ for $15 \mathrm{sec}$ and annealing/extension at $60^{\circ} \mathrm{C}$ for $1 \mathrm{~min}$. The primers were designed and purchased from Sangon Biotech Co., Ltd. (Shanghai, China), and the sequences are shown in Table I. GAPDH was used as a reference gene, and relative gene expression was determined using the $2^{-\Delta \Delta \mathrm{Cq}}$ method (26).

Western blotting. Proteins were extracted from colon tissues using radioimmunoprecipitation assay buffer supplemented with protease inhibitors (Shanghai Shenggong Co., Ltd., Shanghai, China) and protein concentration was measured using the Bicinchoninic Acid Protein Assay kit (Nanjing KeyGen Biotech Co., Ltd., Nanjing, China). Subsequently, proteins $(50 \mu \mathrm{g})$ were separated by $10 \%$ SDS-PAGE and were transferred to a nitrocellulose membrane (Whatman; GE Healthcare Life Sciences, Little Chalfont, UK). The membrane was then blocked with $5 \%$ skimmed milk at room temperature for $1 \mathrm{~h}$ and was incubated overnight at $4^{\circ} \mathrm{C}$ with anti-mGluR7 antibodies $(1: 1,000$, rabbit anti-rat, cat. no. NB-91787; Novus Biologicals, LLC, Littleton, CO, USA) or antibodies against total NF- $\mathrm{B}$ (p65, 1:1,000, rabbit anti-rat,

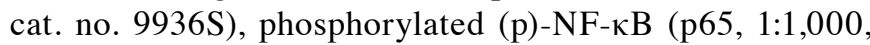
rabbit anti-rat, cat. no. $9936 \mathrm{~S})$ or $\beta$-actin $(1: 1,000$, rabbit anti-rat, cat. no. 8457) (all from Cell Signaling Technology, Inc., Danvers, MA, USA). The membrane was then incubated with an IRDye ${ }^{\circledR} 800 \mathrm{CW}$-conjugated secondary antibody (1:2,000, cat. no. A80-195P; Rockland Immunochemicals, Inc., Limerick, PA, USA) for $1 \mathrm{~h}$ at room temperature. Images were acquired using an Odyssey infrared imaging system (LI-COR Biosciences, Lincoln, NE, USA). The blots were semi-quantified by grey value analysis (ImageJ 1.50i; NIH, Bethesda, MA, USA).

Immunohistochemistry. Colon tissues were fixed in $4 \%$ formalin overnight at $4^{\circ} \mathrm{C}$; subsequently, paraffin-embedded colon tissues (length, $1 \mathrm{~cm}$; width, $0.5 \mathrm{~cm}$ ) were mounted on slides, deparaffinised with xylene (Sinopharm Chemical Reagent Co., Ltd., Shanghai, China) and rehydrated with $1.5 \%$ $\mathrm{H}_{2} \mathrm{O}_{2}$ in PBS for $30 \mathrm{~min}$ at room temperature. Tissues were 
A

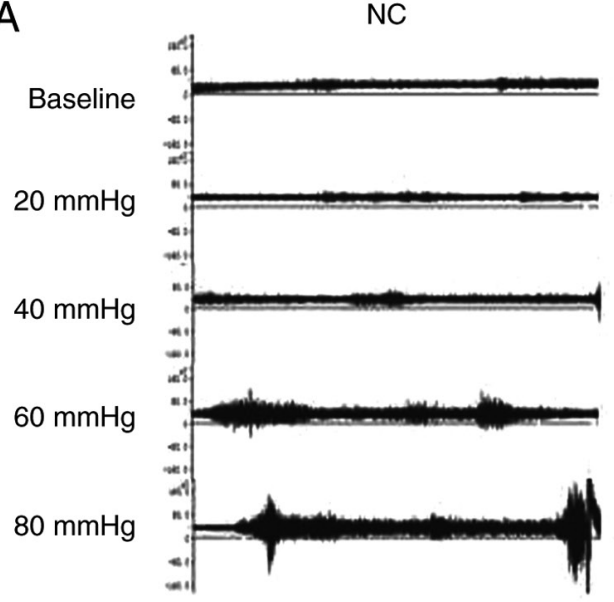

$\mathrm{mmHg} \mid 20 \mathrm{Sec}$
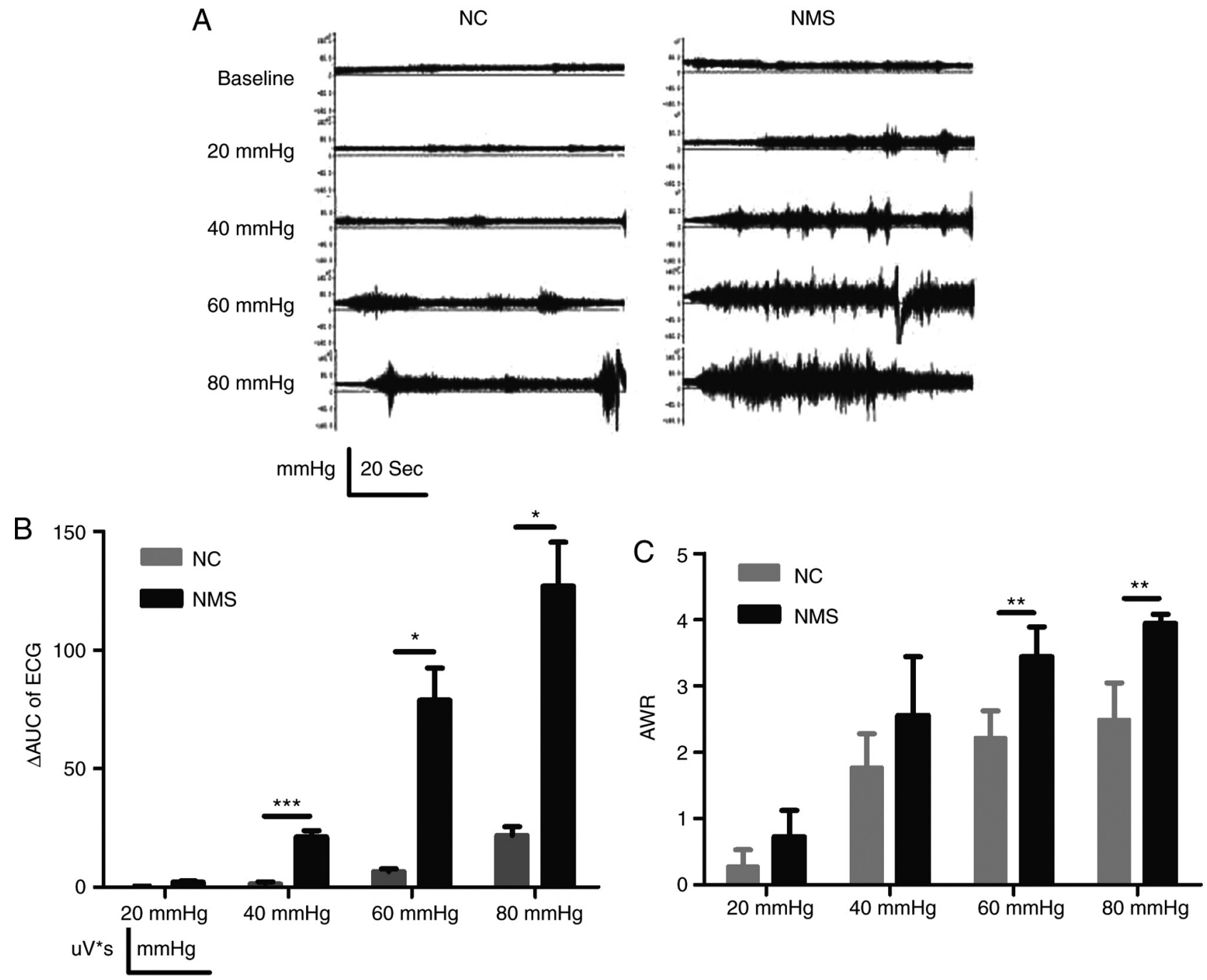

Figure 1. Visceral hypersensitivity of adult male rats. Results were analysed between the NC and NMS groups. (A) EMG signal recordings of an NC rat and an NMS rat in response to CRD. (B) Statistical analysis of the EMG amplitudes of the NC and NMS groups using the $\triangle$ AUC formula (AUC during CRD-AUC before CRD). (C) Statistical analysis of the AWR scores of the NC and NMS groups. Data were analysed by Student's t-test and are expressed as the means \pm standard error of the mean (number of rats $=6$ ). ${ }^{*} \mathrm{P}<0.05^{* *} \mathrm{P}<0.01,{ }^{* * *} \mathrm{P}<0.001$. AUC, area under the curve; AWR, abdominal withdrawal reflex; CRD, colorectal distension; EMG, electromyography; NC, normal control; NMS, neonatal maternal separation.

incubated overnight at $4^{\circ} \mathrm{C}$ with anti-mGluR7 antibody (1:500, rabbit anti-rat, cat. no. NB-91787; Novus Biologicals LLC), or antibodies against cluster of differentiation (CD)3 (1:100, rabbit anti-rat, cat. no. ab16669) CD68 (1:200, mouse anti-rat, cat. no. ab31630) or myeloperoxidase (MPO; 1:50, rabbit anti-rat, cat. no. ab9535) (Abcam, Cambridge, UK). Secondary goat anti-rabbit antibodies or goat anti-mouse antibodies (1:200, cat. nos. A0277 and A0286; Beyotime Institute of Biotechnology, Shanghai, China) were then added to the tissues for $1 \mathrm{~h}$ at room temperature. After being incubated with streptavidin-conjugated horseradish peroxidase (HRP) [Rabbit Specific HRP/DAB (ABC) Detection IHC kit, cat. no. ab64261; Abcam] for 10 min at room temperature, sections were stained with DAB for 1-10 min at room temperature (1:2; Gene Tech Biotechnology Co., Ltd., Shanghai, China); sections were then counterstained with haematoxylin for 0.5-2 $\mathrm{min}$ at room temperature. The sections were visualised under an Olympus BX41-32P02-FLB3 microscope (Olympus Corporation, Tokyo, Japan). Images were captured using FluoView software (FV1000, Olympus Corporation).
Statistical analysis. The experiments were repeated three times, and all data are presented as the means \pm standard error of the mean. Statistical analyses were performed using SPSS 19.0 (IBM SPSS, Armonk, NY, USA). Statistical significance of differences between two groups was determined with Student's t-test, whereas multiple groups were analysed by one-way analysis of variance followed by the least significant difference test or Dunnett's T3 test. $\mathrm{P}<0.05$ was considered to indicate a statistically significant difference.

\section{Results}

Early NMS causes CRD-induced visceral hypersensitivity in rats. Initially, pain threshold in response to CRD was determined using the AWR test and EMG. Electromyogram activity in the NMS group was enhanced compared with in the NC group (Fig. 1A). To quantitatively measure EMG, $\triangle \mathrm{AUC}$ was used. Compared with in the NC group, the $\triangle \mathrm{AUC}$ in the NMS group was significantly increased at CRDs of 40, 60 and $80 \mathrm{mmHg}(\mathrm{P}<0.001, \mathrm{P}<0.05$ and $\mathrm{P}<0.05$, respectively; 
Fig. 1B). AWR in the NMS group was significantly higher compared with in the $\mathrm{NC}$ group at CRDs of 60 and $80 \mathrm{mmHg}$ $(\mathrm{P}<0.01$ and $\mathrm{P}<0.01$, respectively; Fig. $1 \mathrm{C})$. These findings indicated that NMS could simulate negative emotional events during early IBS, and the model successfully induced visceral hypersensitivity.

$m$ GluR7 expression is significantly increased in the colons of rats with visceral hypersensitivity. The expression levels of mGluR7 were increased in the colon tissues of NMS rats compared with in the NC group, as determined by immunohistochemistry. Notably, staining was primarily observed in the mucosa, including a portion of cells at the surface epithelium, as well as in the lamina propria, with particularly strong staining in the bottom of the crypts (Fig. 2A). The mRNA and protein expression levels of mGluR7 were increased in the colon tissues of NMS rats compared with in the NC group $(\mathrm{P}<0.05$; Fig. 2B and $\mathrm{C})$. A significant difference was detected between the two groups $(\mathrm{P}<0.05$; Fig. $2 \mathrm{D})$. These findings indicated that mGluR7 expression was increased in the colons of rats with visceral hypersensitivity; therefore, IBS episodes may be associated with mGluR7 expression.

Selective mGluR7 agonist AMN082 (10 mg/kg) attenuates $C R D$-induced visceral hypersensitivity in NMS rats. The effects of AMN082 on alterations in mGluR7 expression and visceral hypersensitivity were detected. Following treatment with AMN082, the protein expression levels of mGluR7 were increased (Fig. 3A). The electromyogram activity in the NMS + AMN082 (10 mg/kg) group was attenuated compared with in the NMS group (Fig. 3B). Compared with in the NMS group, the $\triangle \mathrm{AUC}$ in the NMS + AMN082 $(10 \mathrm{mg} / \mathrm{kg})$ group was significantly decreased at CRDs of 40,60 and $80 \mathrm{mmHg}(\mathrm{P}<0.01, \mathrm{P}<0.01$ and $\mathrm{P}<0.05$, respectively; Fig. 3C). No significant alteration was observed between the NMS and NMS + AMN082 (3 mg/kg) groups. Furthermore, the AWR in the NMS + AMN082 $(10 \mathrm{mg} / \mathrm{kg})$ group was significantly reduced compared with in the NMS group at CRDs of 60 and $80 \mathrm{mmHg}(\mathrm{P}<0.01$ and $\mathrm{P}<0.05$, respectively; Fig. 3D). At a dose of $3 \mathrm{mg} / \mathrm{kg}$, the AWR results in the NMS + AMN082 group were not significantly different compared with in the NMS group. These findings suggested that $10 \mathrm{mg} / \mathrm{kg}$ AMN082 significantly activated mGluR7 and attenuated CRD-induced visceral hypersensitivity in NMS rats.

AMN082 (10 mg/kg) suppresses the expression levels of pro-inflammatory cytokines and increases the expression levels of anti-inflammatory cytokines in colon tissues of NMS rats. Since $10 \mathrm{mg} / \mathrm{kg}$ AMN082 significantly attenuated CRD-induced visceral hypersensitivity in NMS rats, the present study explored the underlying mechanisms. The mRNA expression levels of the anti-inflammatory cytokines IL-10 and TGF- $\beta 1$, the pro-inflammatory cytokine IFN- $\gamma$, NOS and 5-HTRs were detected in colon tissues by RT-qPCR (Fig. 4). IL-10 was decreased in the NMS group compared with in the NC group $(\mathrm{P}<0.01)$, whereas IFN- $\gamma$ was increased $(\mathrm{P}<0.05)$. Notably, treatment with $10 \mathrm{mg} / \mathrm{kg}$ AMN082 significantly increased the release of IL-10 $(\mathrm{P}<0.01)$ and TGF- $\beta 1$ $(\mathrm{P}<0.01)$, and suppressed the release of IFN- $\gamma(\mathrm{P}<0.05)$. NOS and 5-HTRs exhibited almost no change in expression;
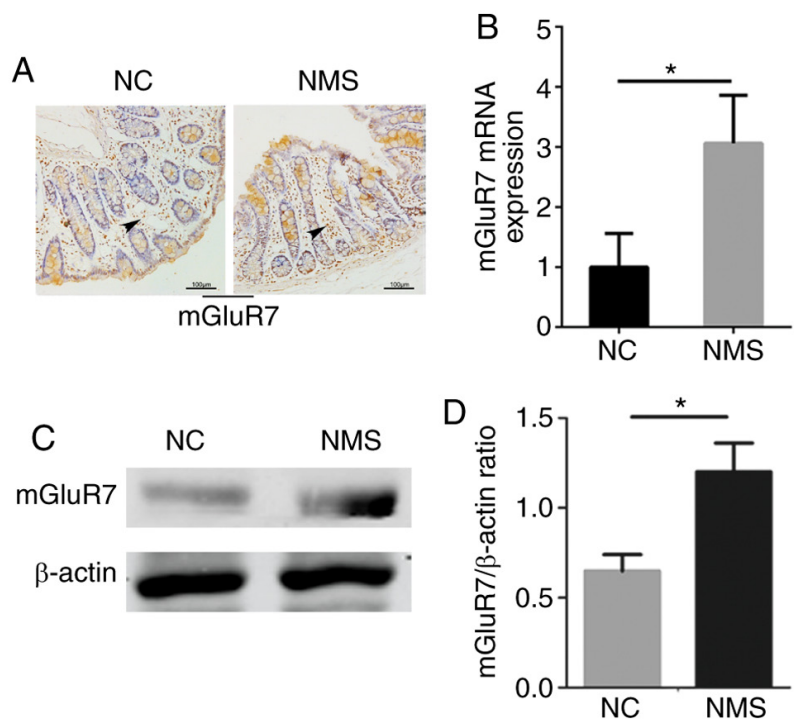

Figure 2. mGluR7 expression in the colon of rats in the NC and NMS groups (A) Immunohistochemistry of mGluR7 in rat colons. Staining in the mucosa (black arrowheads) was compared between the NC and NMS group; there was no difference beyond the submucosa (magnification, x200). (B) mRNA expression levels of mGluR7 in the rat colons. (C) Protein expression levels of mGluR7 in the rat colons. (D) Grey values were used in the semi-quantitative analysis of western blotting results; $\mathrm{mGluR} 7 / \beta$-actin ratio was used to indicate relative protein expression levels of mGluR7. Data were analysed by Student's t-test and are expressed as the means \pm standard error of the mean (number of rats $=6$ ). ${ }^{*} \mathrm{P}<0.05$. mGluR7, metabotropic glutamate receptor-7; $\mathrm{NC}$, normal control; NMS, neonatal maternal separation.

however, iNOS was significantly decreased in the NMS group compared with in the $\mathrm{NC}$ group $(\mathrm{P}<0.05)$. Since the release of inflammatory cytokines is an indicator of the inflammatory response, these results indicated that AMN082 may inhibit inflammation in the colons of NMS rats.

AMN082 (10 $\mathrm{mg} / \mathrm{kg})$ reduces the expression levels of MPO and the number of $\mathrm{CD}^{+}$cells in the intestinal mucosa of $\mathrm{NMS}$ rats. MPO enzymatic activity is an index of neutrophil infiltration in colon tissue; and $\mathrm{CD}^{+}$is a specific membrane surface molecule on T lymphocytes, which is often used as a marker of T lymphocytes; CD68 is a marker of macrophages or monocytes, Both $\mathrm{T}$ cells and macrophages participate in regulating the intestinal immune system $(27,28)$; therefore, MPO, CD3 and CD68 were detected in the present study (Fig. 5). NMS rats exhibited increased MPO expression levels, and CD3 and CD68 infiltration in colon tissue compared with the $\mathrm{NC}$ group, and staining was primarily observed in the mucosa (Fig. 5A, B, D, E, G and H). Notably, CD68 staining was the least obvious. In addition, the NMS + AMN082 $(10 \mathrm{mg} / \mathrm{kg})$ group exhibited markedly decreased $\mathrm{CD}^{+} \mathrm{T}$ cell infiltration compared with the NMS group (Fig. 5B and C). Similarly, administration of $10 \mathrm{mg} / \mathrm{kg}$ AMN082 markedly reduced MPO expression compared with the NMS group (Fig. $5 \mathrm{H}$ and I); there was no difference in CD68 expression in the $\mathrm{NMS}+\mathrm{AMN082}(10 \mathrm{mg} / \mathrm{kg})$ group compared with the NMS group (Fig. 5E and F).

AMN082 (10 mg/kg) inhibits activation of $N F-\kappa B$ in the colon of NMS rats. NF- $\mathrm{BB}$ is a critical transcription factor in the inflammatory response. This protein functions as 


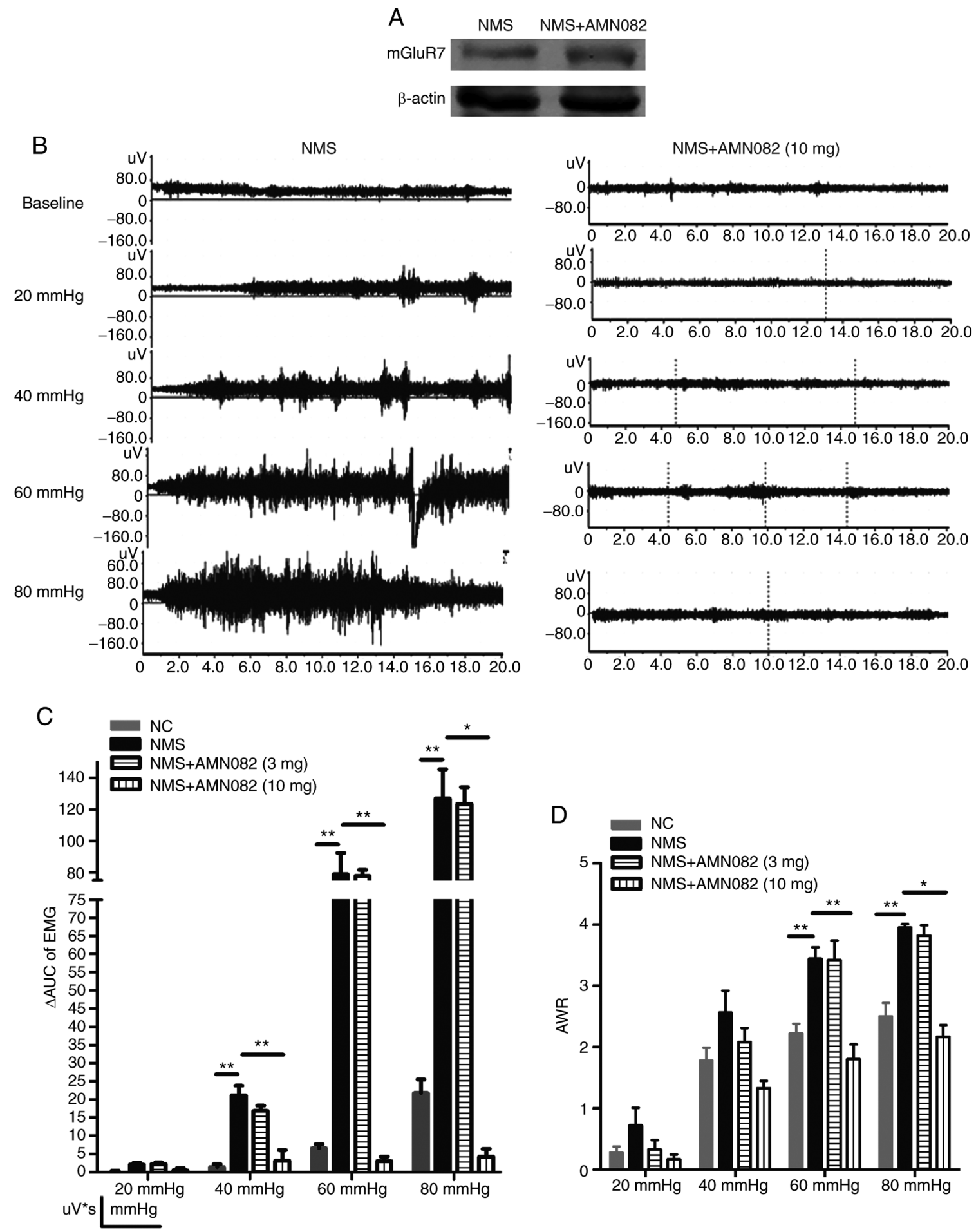

Figure 3. Effects of AMN082 on visceral hypersensitivity in NMS rats. AMN082 (3 or $10 \mathrm{mg} / \mathrm{kg})$ was administered intraperitoneally $1 \mathrm{~h}$ prior to the tests. (A) Protein expression levels of mGluR7 in the colon samples obtained from rats in the NMS and NMS + AMN082 (10 mg/kg) groups. (B) EMG signal recordings of an NMS rat and an NMS + AMN082 (10 mg/kg) rat in response to CRD. (C) Statistical analysis of the EMG amplitude among the groups. (D) Statistical analysis of the AWR scores among the groups. AWR data were analysed by one-way ANOVA and least significant difference test; EMG data were analysed by one-way ANOVA and Dunnett's T3 test. Data are expressed as the means \pm standard error of the mean (number of rats $=6$ ). ${ }^{*} \mathrm{P}<0.05,{ }^{* *} \mathrm{P}<0.01$. ANOVA, analysis of variance; AUC, area under the curve; AWR, abdominal withdrawal reflex; CRD, colorectal distension; EMG, electromyography; mGluR7, metabotropic glutamate receptor-7; NMS, neonatal maternal separation.

a pro-inflammatory factor that participates in the pathophysiology of intestinal inflammatory diseases (29). Intestinal inflammation may provide an initial stimulus for a persistent state of visceral hypersensitivity (30). To investigate the mechanism underlying the anti-inflammatory activity of AMN082, the effects of AMN082 on activation of the NF- $\kappa$ B pathway in rats were investigated. Alterations in the expression levels of $\mathrm{p}-\mathrm{NF}-\kappa \mathrm{B}$ p65 in the colons of NMS rats were evaluated by western blotting (Fig. 6A); p-NF- $\mathrm{B}$ p65 was upregulated in NMS rats compared with in the NC group. Conversely, 


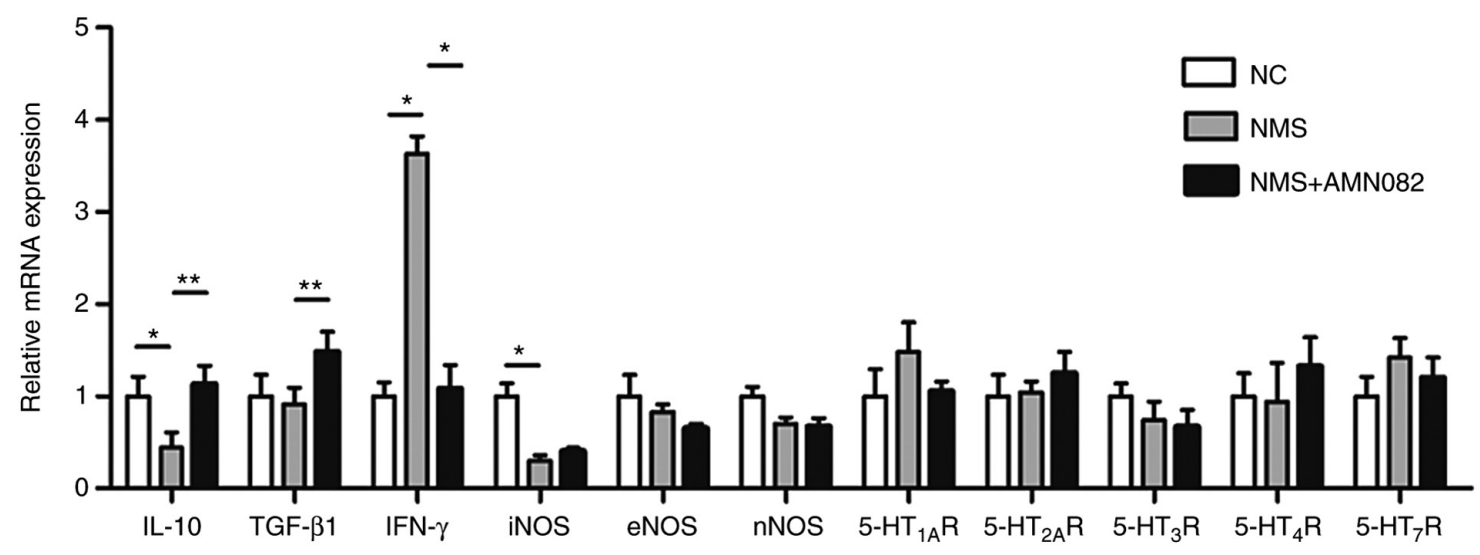

Figure 4. Effects of AMN082 (10 mg/kg) on the mRNA expression levels of 5-HT receptors, NOS and inflammatory cytokines. IL-10 and TGF- $\beta 1$ data were analysed by one-way ANOVA and least significant difference test; iNOS and IFN- $\gamma$ data were analysed by one-way ANOVA and Dunnett's T3 test. Data are expressed as the means \pm standard error of the mean (number of rats $=6$ ). ${ }^{*} \mathrm{P}<0.05,{ }^{* *} \mathrm{P}<0.01 .5$ - $\mathrm{HT}$, 5-hydroxytryptamine; 5-HT ${ }_{1 \mathrm{~A}} \mathrm{R}, 5-\mathrm{HT} 1 \mathrm{~A}$ receptor; 5- $\mathrm{HT}_{2 \mathrm{~A}} \mathrm{R}, 5-\mathrm{HT} 2 \mathrm{~A}$ receptor; 5- $\mathrm{HT}_{3} \mathrm{R}, 5-\mathrm{HT} 3$ receptor; 5-HT $\mathrm{R}$, 5-HT 4 receptor; 5-HT 7 R, 5-HT 7 receptor; ANOVA, analysis of variance; eNOS, endothelial nitric oxide synthase; IFN- $\gamma$, interferon- $\gamma$; IL-10, interleukin-10; iNOS, inducible nitric oxide synthase; NC, normal control; NMS, neonatal maternal separation; nNOS, neuronal nitric oxide synthase; TGF- $\beta 1$, transforming growth factor- $\beta 1$.
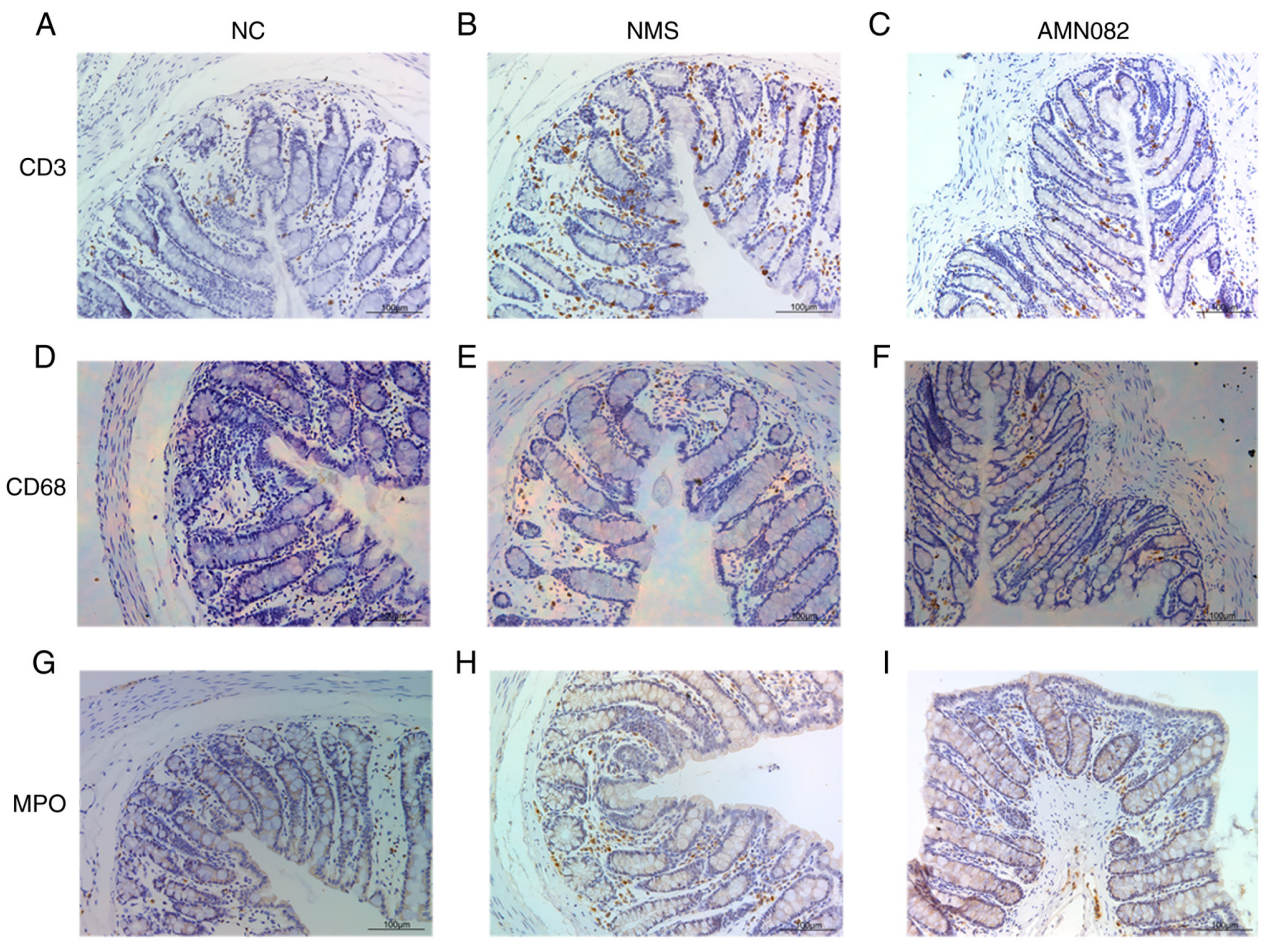

Figure 5. Effects of AMN082 $(10 \mathrm{mg} / \mathrm{kg})$ on inflammatory infiltration into the colon mucosa. The infiltration of neutrophils, macrophage cells and CD $3^{+} \mathrm{T}$ cells was detected by immunohistochemistry in the colons of rats in the NC, NMS and NMS + AMN082 (10 mg/kg) groups. (A-C) CD3 staining; (D-F) CD68 staining; and (G-I) MPO staining (magnification, x200). Positive cells were stained yellow or brown-yellow, and the distribution of positive cells in the colon was mainly localised in the lamina propria of the mucosa and scattered around the intestinal gland. CD, cluster of differentiation; MPO, myeloperoxidase; $\mathrm{NC}$, normal control; NMS, neonatal maternal separation.

AMN082 treatment significantly reduced $\mathrm{p}-\mathrm{NF}-\mathrm{\kappa B}$ expression compared with in the NMS group (Fig. 6B; $\mathrm{P}<0.05$ ).

\section{Discussion}

The present study demonstrated that early NMS induced visceral hypersensitivity in rats and increased the expression of mGluR7 in the colon. However, selective activation of mGluR7 via AMN082 $(10 \mathrm{mg} / \mathrm{kg})$ attenuated CRD-induced visceral hypersensitivity. In addition, AMN082 modulated the immune response in NMS rats by regulating the balance between pro-inflammatory factors and anti-inflammatory factors, and suppressing low-grade inflammation via the reduction of NF- $\mathrm{\kappa B}$ activation.

A strong body of evidence supports the role of glutamate as a primary neurotransmitter in the vagal circuitry, which is involved in key gastrointestinal functions (31). In addition, mGluRs appear to be relevant not only for the modulation of gastrointestinal vagovagal reflexes, but also for the process of digestion as a whole (8). mGluR4 has been detected in 

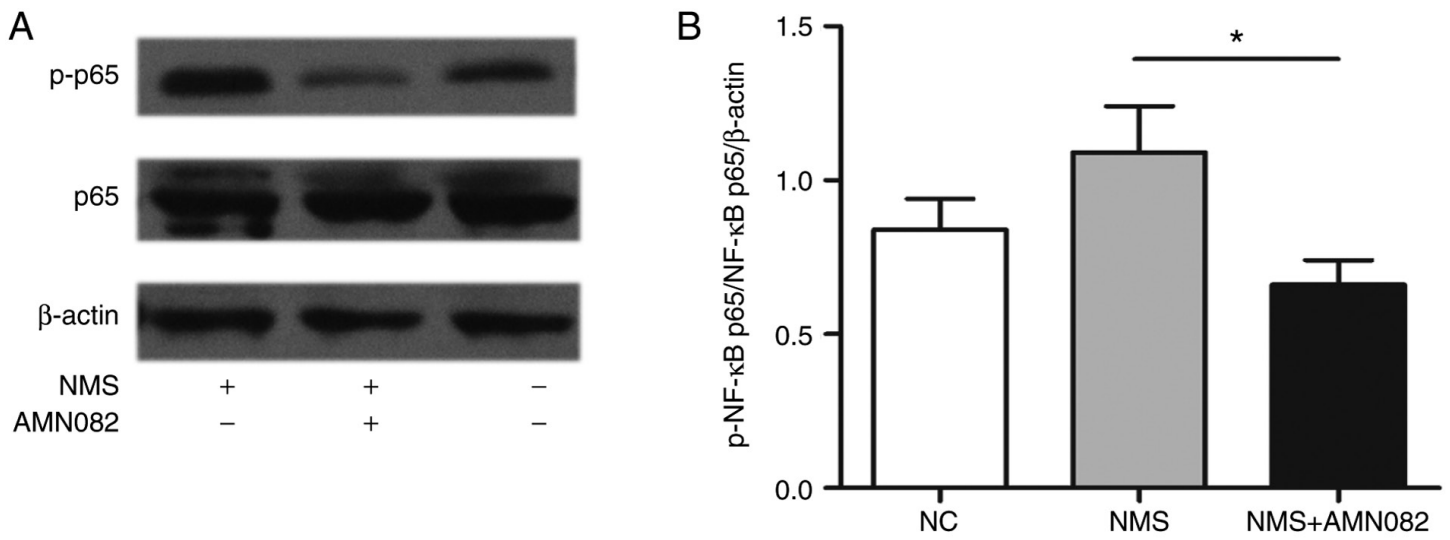

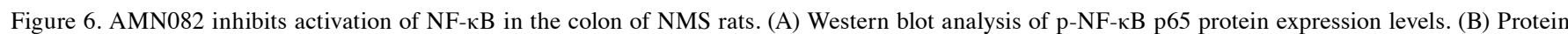
expression levels of p-NF-кB p65 were semi-quantified by grey value analysis. Data were analysed by one-way analysis of variance followed by least significant difference test. Data are expressed as the means \pm standard error of the mean (number of rats $=6$ ). $\mathrm{P}<0.05$. NC, normal control; NF- $\mathrm{\kappa B}$, nuclear factor- $\mathrm{kB}$; NMS, neonatal maternal separation; p-, phosphorylated.

normal human colon epithelium (32), and an accelerating effect on guinea pig colon motility and longitudinal muscle contractions has been observed upon application of mGluR8 agonists on isolated tissue (33). Furthermore, mGluR7 mRNA and protein are expressed in the mouse colon mucosa, and treatment with the selective mGluR7 agonist AMN082 induces an increase in faecal water content in stress-induced defecation (6). Previous evidence has indicated that overexpression of the N-methyl-D-aspartate receptor (an ionotropic receptor) serves an important role in the formation of post-inflammatory visceral hypersensitivity (34). Therefore, it was hypothesised that mGluR7 may be relevant to visceral hypersensitivity. The majority of studies regarding IBS and AMN082 (mGlu7 receptor agonist) have used animal models; AMN082 is active in the central nervous system via direct central injection, and is also active outside of the central nervous system via oral or intraperitoneal injection $(6,35,36)$. The present study demonstrated that mGluR7 expression was enhanced in NMS rats, as determined by assessing immunohistochemistry, mRNA levels and protein levels. Therefore, it was suggested that mGluR7 may have an important role in the visceral hypersensitivity of IBS; these results were similar to those of Julio-Pieper et al (6). A high frequency of IBS symptoms has been detected in patients with panic disorder, generalised anxiety disorder and major depressive disorder, and another study reported that mGluR7-knockout animals display an anxious phenotype (8). The present study confirmed that activating mGluR7 attenuated CRD-induced visceral hypersensitivity. This conclusion was consistent with previous literature reports, and represented a breakthrough in understanding the association between mGluR7 and the pathogenesis of IBS.

It has been reported that the abnormal perception of visceral stimuli, or visceral hypersensitivity, is an important underlying mechanism of IBS, and intestinal inflammation may provide an initial stimulus for a persistent state of visceral hypersensitivity (31). Patients with post-infectious IBS exhibit no signs of overt inflammation but exhibit persistent minor increases in epithelial $\mathrm{T}$ lymphocytes and mast cells (37), thus suggesting that long-term inflammatory alterations may be responsible for colonic hypersensitivity. Furthermore, it has been indicated that a $\mathrm{T}$ helper (Th)1/Th2 immune imbalance is closely associated with patients with IBS with diarrhoea (D-IBS); IFN- $\gamma$ is a Th1-mediated cytokine that promotes the inflammatory response, whereas IL-10 is a Th2-mediated cytokine that inhibits the inflammatory response (38). In IL-10-deficient mice, the majority of animals suffer from chronic enterocolitis, thus suggesting that IL-10 is an essential immunoregulator in the intestinal tract (39). TGF- $\beta 1$ is one of the most common members of the TGF- $\beta$ family, which possesses an anti-inflammatory effect. Furthermore, NF- $\kappa B$ is an important transcription factor that is mainly involved in inflammatory and immune responses. NF- $\mathrm{kB}$-dependent inflammatory mediators, including IL-6, are associated with the maternal separation model of IBS, and the downstream activation of extracellular signal-regulated kinase (ERK), Janus kinase (JAK)-signal transducer and activator of transcription (STAT) and NF- $\kappa \mathrm{B}$ signalling cascades (40). Furthermore, stimulating the production of pro-inflammatory cytokines, including IL-1 $\beta$ and tumour necrosis factor (TNF)- $\alpha$, contributes to visceral hypersensitivity through the Toll-like receptor 4 (TLR4)/myeloid differentiation primary response $88 / \mathrm{NF}-\kappa \mathrm{B}$ signalling pathway in the spinal cord in a neonatal colonic irritation rat model (41). In addition, NO is involved in inhibition of the transmission and perception of visceral hypersensitivity (42). A previous study revealed that ioglitazone (hypoglycemic drug) reduces visceral hypersensitivity, increases nociceptive thresholds, NO production and iNOS activity in D-IBS rats (17). In the present study, iNOS expression was decreased in the NMS group, which could further reduce the synthesis of NO, resulting in enhanced intestinal motility and increased visceral hypersensitivity. Similar to the aforementioned findings, a decrease in iNOS expression may be associated with the occurrence of visceral hypersensitivity in IBS.

In the present study, decreased IFN- $\gamma$, and increased TGF- $\beta 1$ and IL-10 levels were observed in the intestinal mucosa of AMN082-treated rats. Furthermore, the expression levels of CD3 and MPO were decreased in the intestinal mucosa of AMN082-treated rats. These results indicated that activating mGluR7 may result in an imbalance of Th1/Th2 immunity. Upregulated IL-10 reduces antigen-specific human 
T-cell proliferation by diminishing the antigen-presenting capacity of monocytes (43), which then inhibits the secretion of TNF- $\alpha$ and results in the inhibition of $N F-\kappa B$ pathway activation. A previous report suggested that during acute infection of IBS, TGF- $\beta 1$ is increased in the muscle layer (44); therefore, in AMN082-treated NMS rats, upregulated TGF- $\beta 1$ may inhibit the maturation of dendritic cells and Th1 activity, and enhance Th2 activity. Conversely, downregulated IFN- $\gamma$ can reduce the activation of macrophages and inhibit Th1 activity, also resulting in the inhibition of $\mathrm{NF}-\kappa \mathrm{B}$ pathway activation. The decreased expression of $\mathrm{NF}-\kappa \mathrm{B}$ in AMN082-treated NMS rats is consistent with the aforementioned conclusions. When $N F-\kappa B$ is activated various downstream inflammatory cytokines are produced. These findings indicated that activating mGluR7 may reduce $\mathrm{NF}-\kappa \mathrm{B}$-associated amplification of the inflammatory response, thereby protecting the intestinal mucosal barrier and reducing intestinal permeability. Subsequently, the number of antigens that pass through the intestine and immune cell interactions are reduced. When the initial inflammatory stimulus is weakened, the persistent state of visceral hypersensitivity may be relieved.

The present study did not assess the mGluR7 signalling pathway in vitro; therefore, further studies may be conducted using a cell model and mGluR7 small interfering RNA. To examine the effects of mGluR7 more detail, it would be beneficial to determine the effects of mGluR7 gene knockdown or antagonism in rats. In addition, the association between mGluR7 and other characteristics of IBS, such as diarrhoea and constipation, should be evaluated; in these future studies, stool characteristics, including stool particles and faecal water content, may be detected. Other IBS-associated inflammatory signalling pathways, including ERK, JAK-STAT, TLR4 and $\mathrm{NF}-\kappa \mathrm{B}$ signalling cascades $(40,41)$, may also have a relationship with altered mGluR7 in rats with visceral hypersensitivity; further studies are required to evaluate this.

Stressful life events and experimental stress exacerbate symptoms and visceral hypersensitivity in patients with functional gastrointestinal disorders, such as IBS (13). In conclusion, the present study supported the idea of a complex interaction between the gastrointestinal tract and the brain, namely, the brain-gut axis. The present data demonstrated that mGluR7 may have an important role in attenuating visceral hypersensitivity in IBS, in addition to regulating the central components of chronic stress, and regulating fluid and electrolyte transport in the intestine. The mechanisms underlying these effects may be associated with inhibition of $\mathrm{NF}-\kappa \mathrm{B}$ activation and reduced inflammation.

\section{Acknowledgements}

Not applicable.

\section{Funding}

No funding was received.

\section{Availability of data and material}

The datasets used and/or analysed during the current study are available from the corresponding author on reasonable request.

\section{Authors' contributions}

LMS and YBL were involved in the conception and design, and conducted most of the study. JHX and QYW assisted with the immunohistochemistry, western blotting and RT-qPCR experiments. LMS was involved in the acquisition, analysis and interpretation of data, and drafted the article. FL and JD made contributions to the conception and design of the study, and analysed and interpreted the data; they were also involved in revising the manuscript critically for important intellectual content and gave final approval of the version to be published. All authors participated sufficiently in the work to take public responsibility for appropriate portions of the content and agreed to be accountable for all aspects of the work. The final manuscript was read and approved by all authors.

\section{Ethics approval and consent to participate}

All animal experiments were performed following approval by the Animal Care and Use Committee of Tongji University School of Medicine.

\section{Patient consent for publication}

Not applicable.

\section{Competing interests}

The authors declare that they have no competing interests.

\section{References}

1. Zhao K, Yu L, Wang X, He Y and Lu B: Clostridium butyricum regulates visceral hypersensitivity of irritable bowel syndrome by inhibiting colonic mucous low grade inflammation through its action on NLRP6. Acta Biochim Biophys Sin (Shanghai) 50: 216-223, 2018.

2. Rodiño-Janeiro BK, Vicario M, Alonso-Cotoner C, PascuaGarcía R and Santos J: A review of microbiota and irritable bowel syndrome: Future in therapies. Adv Ther 35: 289-310, 2018.

3. Tjong YW, Ip SP, Lao L, Wu J, Fong HH, Sung JJ, Berman B and Che CT: Role of neuronal nitric oxide synthase in colonic distension-induced hyperalgesia in distal colon of neonatal maternal separated male rats. Neurogastroenterol Motil 23: e666-e278, 2011.

4. Coutinho SV, Plotsky PM, Sablad M, Miller JC, Zhou H, Bayati AI, McRoberts JA and Mayer EA: Neonatal maternal separation alters stress-induced responses to viscerosomatic nociceptive stimuli in rat. Am J Physiol Gastrointest Liver Physiol 282: G307-G316, 2002.

5. Fernández-Montoya J, Avendaño C and Negredo P: The glutamatergic system in primary somatosensory neurons and its involvement in sensory input-dependent plasticity. Int J Mol Sci 19: E69, 2017.

6. Julio-Pieper M, Hyland NP, Bravo JA, Dinan TG and Cryan JF: A novel role for the metabotropic glutamate receptor-7: Modulation of faecal water content and colonic electrolyte transport in the mouse. Br J Pharmacol 160: 367-375, 2010.

7. Golubeva AV, Moloney RD, O'Connor RM, Dinan TG and Cryan JF: Metabotropic glutamate receptors in central nervous system diseases. Curr Drug Targets 17: 538-616, 2016.

8. Julio-Pieper M, O'Connor RM, Dinan TG and Cryan JF: Regulation of the brain-gut axis by group III metabotropic glutamate receptors. Eur J Pharmacol 698: 19-30, 2013.

9. Kłak K, Pałucha A, Brański P, Sowa M and Pilc A: Combined administration of PHCCC, a positive allosteric modulator of mGlu4 receptors and ACPT-I, mGlu III receptor agonist evokes antidepressant-like effects in rats. Amino Acids 32: 169-172, 2007. 
10. Stachowicz K, Brañski P, Kłak K, van der Putten H, Cryan JF, Flor PJ and Andrzej P: Selective activation of metabotropic G-protein-coupled glutamate 7 receptor elicits anxiolytic-like effects in mice by modulating GABAergic neurotransmission. Behav Pharmacol 19: 597-603, 2008.

11. Al-Chaer ED, Kawasaki M and Pasricha PJ: A new model of chronic visceral hypersensitivity in adult rats induced by colon irritation during postnatal development. Gastroenterology 119 : 1276-1285, 2000.

12. Palucha A, Klak K, Branski P, van der Putten H, Flor PJ and Pilc A: Activation of the mGlu7 receptor elicits antidepressant-like effects in mice. Psychopharmacology (Berl) 194: 555-562, 2007.

13. Shah E, Rezaie A, Riddle M and Pimentel M: Psychological disorders in gastrointestinal disease: Epiphenomenon, cause or consequence? Ann Gastroenterol 27: 224-230, 2014.

14. Gros DF, Antony MM, McCabe RE and Swinson RP: Frequency and severity of the symptoms of irritable bowel syndrome across the anxiety disorders and depression. J Anxiety Disord 23: 290-296, 2009.

15. Yan C, Xin-Guang L, Hua-Hong W, Jun-Xia L and Yi-Xuan L: Effect of the 5-HT4 receptor and serotonin transporter on visceral hypersensitivity in rats. Braz J Med Biol Res 45: 948-954, 2012.

16. Hoffman JM, Tyler K, MacEachern SJ, Balemba OB, Johnson AC, Brooks EM, Zhao H, Swain GM, Moses PL, Galligan JJ, et al: Activation of colonic mucosal 5-HT(4) receptors accelerates propulsive motility and inhibits visceral hypersensitivity. Gastroenterology 142: 844-854 e844, 2012.

17. Paragomi P, Rahimian R, Kazemi MH, Gharedaghi $\mathrm{MH}$, Khalifeh-Soltani A, Azary S, Javidan AN, Moradi K, Sakuma S and Dehpour AR: Antinociceptive and antidiarrheal effects of pioglitazone in a rat model of diarrhoea-predominant irritable bowel syndrome: Role of nitric oxide. Clin Exp Pharmacol Physiol 41: 118-126, 2014

18. Baker RG, Hayden MS and Ghosh S: NF- $\mathrm{B}$, inflammation, and metabolic disease. Cell Metab 13: 11-22, 2011.

19. Gao J: Correlation between anxiety-depression status and cytokines in diarrhea-predominant irritable bowel syndrome. Exp Ther Med 6: 93-96, 2013.

20. National Research Council: Guide for the Care and Use of Laboratory Animals. The National Academies Press, Washington, DC, 1996.

21. van den Wijngaard RM, Stanisor OI, van Diest SA, Welting O, Wouters MM, Cailotto C, de Jonge WJ and Boeckxstaens GE: Susceptibility to stress induced visceral hypersensitivity in maternally separated rats is transferred across generations. Neurogastroenterol Motil 25: e780-e790, 2013.

22. Li L, Xie R, Hu S, Wang Y, Yu T, Xiao Y, Jiang X, Gu J, Hu CY and $\mathrm{Xu}$ GY: Upregulation of cystathionine beta-synthetase expression by nuclear factor-kappa B activation contributes to visceral hypersensitivity in adult rats with neonatal maternal deprivation. Mol Pain 8: 89, 2012.

23. Matsumoto K, Takagi K, Kato A, Ishibashi T, Mori Y, Tashima K, Mitsumoto A, Kato S and Horie S: Role of transient receptor potential melastatin 2 (TRPM2) channels in visceral nociception and hypersensitivity. Exp Neurol 285: 41-50, 2016.

24. van den Wijngaard RM, Stanisor OI, van Diest SA, Welting O, Wouters MM, de Jonge WJ and Boeckxstaens GE: Peripheral $\alpha$-helical CRF (9-41) does not reverse stress-induced mast cell dependent visceral hypersensitivity in maternally separated rats. Neurogastroenterol Motil 24: 274-282, e111, 2012.

25. Li X, Xi ZX and Markou A: Metabotropic glutamate 7 (mGlu7) receptor: A target for medication development for the treatment of cocaine dependence. Neuropharmacology 66: 12-23, 2013.

26. Livak KJ and Schmittgen TD: Analysis of relative gene expression data using real-time quantitative PCR and the 2(-Delta Delta C(T)) method. Methods 25: 402-408, 2001.

27. Xiao J, Shao L, Shen J, Jiang W, Feng Y, Zheng P and Liu F: Effects of ketanserin on experimental colitis in mice and macrophage function. Int J Mol Med 37: 659-668, 2016.
28. Xiao J, Lu Z, Sheng J, Song Y, Jiang W, Liu F and Zheng P 5-fluorouracil attenuates dextran sodium sulfate-induced acute colitis in mice. Mol Med Rep 13: 2821-2828, 2016.

29. Karrasch T and Jobin C: NF-kappaB and the intestine: Friend or foe? Inflamm Bowel Dis 14: 114-124, 2008.

30. Wouters MM: Histamine antagonism and postinflammatory visceral hypersensitivity. Gut 63: 1836-1837, 2014.

31. Hornby PJ: Receptors and transmission in the brain-gut axis. II. Excitatory amino acid receptors in the brain-gut axis. Am J Physiol Gastrointest Liver Physiol 280: G1055-G1060, 2001.

32. Chang HJ, Yoo BC, Lim SB, Jeong SY, Kim WH and Park JG: Metabotropic glutamate receptor 4 expression in colorectal carcinoma and its prognostic significance. Clin Cancer Res 11: $3288-3295,2005$

33. Tong Q and Kirchgessner AL: Localization and function of metabotropic glutamate receptor 8 in the enteric nervous system. Am J Physiol Gastrointest Liver Physiol 285: G992-G1003, 2003.

34. Liu Z, Gao J and Wei L: Changes of expressions of NR2A and NR2B in anterior cingulate cortex of post-inflammatory visceral hypersensitive rats. Chin J Gastroenteml 15: 330-334, 2010 (In Chinese).

35. Pałucha-Poniewiera A, Szewczyk B and Pilc A: Activation of the mTOR signaling pathway in the antidepressant-like activity of the mGlu5 antagonist MTEP and the mGlu7 agonist AMN082 in the FST in rats. Neuropharmacology 82: 59-68, 2014.

36. Wang WY, Wang H, Luo Y, Jia LJ, Zhao JN, Zhang HH, Ma ZW, Xue QS and Yu BW: The effects of metabotropic glutamate receptor 7 allosteric agonist $\mathrm{N}, \mathrm{N}$ '-dibenzhydrylethane-1,2-diamine dihydrochloride on developmental sevoflurane neurotoxicity: Role of extracellular signal-regulated kinase 1 and 2 mitogen-activated protein kinase signaling pathway. Neuroscience 205: 167-177, 2012

37. Ohman L and Simrén M: Pathogenesis of IBS: Role of inflammation, immunity and neuroimmune interactions. Nat Rev Gastroenterol Hepatol 7: 163-173, 2010.

38. Li YQ, Zhang HN, Zuo XL, Yuan HP, Lu XF and Li MJ: Study on the shifting of Thl/Th2 balance of large intestinal mueosa in patients with irritable bowel syndrome. Chin J of Dig 24: 728-731, 2004 (In Chinese).

39. Kühn R, Löhler J, Rennick D, Rajewsky K and Müller W: Interleukin-10-deficient mice develop chronic enterocolitis. Cell 75: 263-274, 1993.

40. O'Malley D, Liston M, Hyland NP, Dinan TG and Cryan JF: Colonic soluble mediators from the maternal separation model of irritable bowel syndrome activate submucosal neurons via an interleukin-6-dependent mechanism. Am J Physiol Gastrointest Liver Physiol 300: G241-G252, 2011.

41. Chen ZY, Zhang XW, Yu L, Hua R, Zhao XP, Qin X and Zhang YM: Spinal toll-like receptor 4-mediated signalling pathway contributes to visceral hypersensitivity induced by neonatal colonic irritation in rats. Eur J Pain 19: 176-186, 2015.

42. Rodella L, Rezzani R, Agostini C and Bianchi R: Induction of NADPH-diaphorase activity in the rat periaqueductal gray matter after nociceptive visceral stimulation. Brain Res 793: 333-336, 1998.

43. de Waal Malefyt R, Haanen J, Spits H, Roncarolo MG, te Velde A, FigdorC,Johnson K,Kastelein R, Yssel Hand de VriesJE:Interleukin 10 (IL-10) and viral IL-10 strongly reduce antigen-specific human $\mathrm{T}$ cell proliferation by diminishing the antigen-presenting capacity of monocytes via downregulation of class II major histocompatibility complex expression. J Exp Med 174: 915-924, 1991.

44. Akiho H, Deng Y, Blennerhassett P, Kanbayashi $H$ and Collins SM: Mechanisms underlying the maintenance of muscle hypercontractility in a model of postinfective gut dysfunction. Gastroenterology 129: 131-141, 2005.

This work is licensed under a Creative Commons Attribution-NonCommercial-NoDerivatives 4.0 International (CC BY-NC-ND 4.0) License. 